\title{
Ionospheric Doppler measurements by means of HF-radar techniques
}

\author{
Cesidio Bianchi $\left({ }^{1}\right)$ and David Altadill $\left({ }^{2}\right)$ \\ (') Istituto Nazionale di Geofisica e Vulcanologia, Roma, Italy \\ (') Observatori de l'Ebre, Roquetes, Spain
}

\begin{abstract}
Studies of the dynamics of the ionosphere and its related phenomena are mainly based on Doppler Drift measurements. The time variation (ionisation/recombination) of plasma density, thermospheric wind and others can be observed by means of HF-radars. The technique of Doppler Drift measurements is a quite complex technique that is now affordable by means of an advanced ionospheric sounder. The combination of vertical sounding and interferometric Doppler detection discloses the Doppler sources. The echo signal contains the Doppler shift in frequency imposed on the wave carrier by each point source where the signal is reflected. Other phenomena like environmental noise and the intrinsic error of the measurements that, together with the change in time of the refractive index, affect the measurements in various ways impeding to better quantify the results.
\end{abstract}

Key words shift Doppler-ionosphere - plasma drift

\section{Introduction}

The measurement techniques of Drift Doppler follow the ionospheric vertical sounding where the return signal is analysed in frequency domain. A wave carrier of angular frequency $\omega$ is sent toward to the ionosphere where it is reflected. In the frequency range of $2-15 \mathrm{MHz}$ the solid beam angle of the employed antenna is very wide and the gain as low as 1-3 dB depending on the frequency so that an area of hundreds of square kilometers is illuminated. Because of the rippled ionospheric surfaces and the volume inhomogeneities the signal is reflected back from various point sources that satisfy the reflection law. These point sources if moving are considered Doppler sources that su-

Mailing address: Dr. Cesidio Bianchi, Istituto Nazionale di Geofisica e Vulcanologia, Via di Vigna Murata 605, 00143 Roma, Italy; e-mail: bianchi@ingv.it perimpose a Doppler shift $\left(\Delta \omega_{1}, \Delta \omega_{2}, \ldots\right)$ on the signal. In first approximation the angular pulsation shift $\Delta \omega$ is

$$
\Delta \omega=-2 \omega V / c
$$

where $V$ is the radial velocity of the reflecting point, $c$ is the light velocity. Dividing by $2 \pi$ both side of the above equation and replacing $\omega$ with $c \cdot k$ and $2 \pi f$ we obtain

$$
\Delta f_{D}=-\boldsymbol{k} V / \pi
$$

where, $f$ is the frequency, $\Delta f_{D}$ is the frequency shift due to the Doppler effect, and $\boldsymbol{k}$ wave vector (Bibl et al., 1975; Hunsucker, 1991).

The moving source $(s)$ assigns a shift in frequency according with the above equations and in the antenna we receive a composite signal with different $\Delta \omega_{s}$ that are the contribution of the $s$ significant sources. In case of a single receiving antenna the spectral analysis (typically a complex FFT) furnishes all the spectral components and the related phases and cannot discriminate the spatial distribution of the different sources. In case of 3 or more antennas it is pos- 
sible with a interferometric Doppler technique to resolve the spatial distribution of the significant sources (Davies, 1990).

\section{Principle of Doppler interferometry}

In principle the interferometric Doppler techniques rely on the frequency and phase analysis of the signal picked-up from spaced antennas lying in a plane at appropriate distance. In our description we refer to 4 antennas placed in the geometric barycentre and in vertexes of an equilateral triangle as in fig. 1. In order to better discriminate the phase contributions it is important to maximize the triangle size (more than $50 \mathrm{~m}$ ) and to eliminate the phase ambiguity the distances between the antenna in the centre of the triangle must not be more than the wavelength $\lambda$ employed in the sounding system.

Now, as in ionospheric vertical sounding, suppose a Radio Frequency (RF) pulse is sent to illuminate a large area, the various ionospheric moving isodensity surfaces reflect the RF signal with a superimposed shift Doppler. A

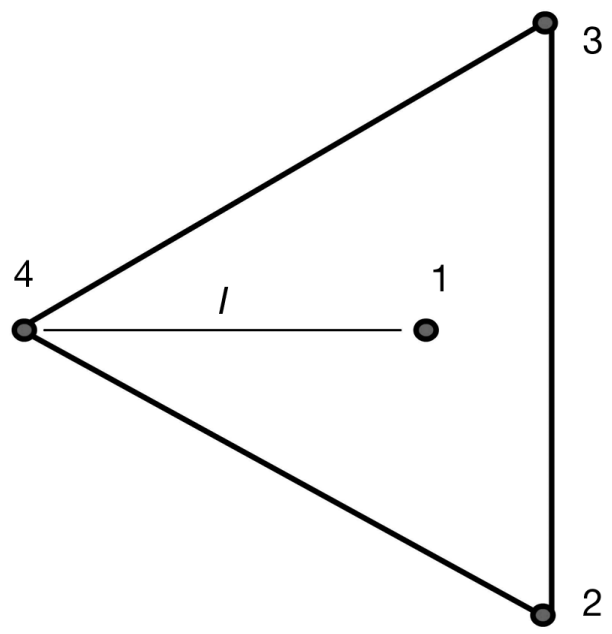

Fig. 1. The receiving antenna at the vertexes and at the geometrical barycentre of an equilateral triangle in an horizontal plane. All the antennas are a the distance $l$. single reflector $s$ contributes with an echo signal that in the receiving antenna 1 has an amplitude $A$ given by

$$
A_{1}=A_{01} \cos \left[\left(\omega+\Delta \omega_{s}\right) t+\phi_{1 s}\right]
$$

where, $A_{01}$ and $\phi_{1 s}$ are respectively the maximum amplitude and the phase at the receiving antenna 1. For the generic antenna $a$ the time varying amplitude will be

$$
A_{a}=A_{0 a} \cos \left[\left(\omega+\Delta \omega_{s}\right) t+\phi_{a s}\right] .
$$

The phase term due to the source $s$ in the antenna 1 is

$$
\phi_{1 s}=\boldsymbol{k}_{s} \cdot \boldsymbol{r}_{1 s}+\delta
$$

where $\boldsymbol{k}$ is the wave vector, $\boldsymbol{r}_{1 s}$ is the oriented vector for the antenna 1 and the source $s$, and $\delta$ is the phase value at the level of the source $s$. If we assume as reference point antenna 1, the phase for the generic antenna $a$ is

$$
\phi_{a s}=\boldsymbol{k}_{s} \cdot \boldsymbol{r}_{1 s}+\delta+\boldsymbol{k}_{s} \cdot \boldsymbol{l}_{a}=\phi_{1 s}+\boldsymbol{k}_{s} \cdot \boldsymbol{l}_{a}
$$

where $\boldsymbol{l}_{a}$ is the oriented vector from antenna 1 and antenna $a$. It means that the phase differences between antenna 1 and the generic antenna $a$ is $\boldsymbol{k}_{s} \cdot \boldsymbol{l}_{a}$.

It must be also noted that because of the distance the $\boldsymbol{r}_{1 s}$ compared with the distance between the antennas the vector $\boldsymbol{k}_{s}$ has the orientation in all the antenna points.

Referring to fig. 2 the phase difference between antenna 1 and the generic antenna $a$ is

$\phi_{\text {las }}=\boldsymbol{k}_{s} \cdot \boldsymbol{l}_{a}=k_{s} \cdot l_{a} \cos (\theta)=2 \pi / \lambda l \cos (\theta)$

where $\theta$ is the angle between $\boldsymbol{k}_{s}$ and $\boldsymbol{l}_{a}$. For a given source that the phase difference between the antenna 1 and the generic antenna $a$, according with the above equation, is a function of the angle $\theta$ between the vector $\boldsymbol{k}$ and $\boldsymbol{l}$. It is worth noting that different Doppler sources are distinguishable by different $\Delta \omega_{s}$ values (where $s$ is the number of sources). For a given Doppler source $\Delta \omega$, once the phase difference is measured knowing the range $r$, the wavelength $\lambda$ and 


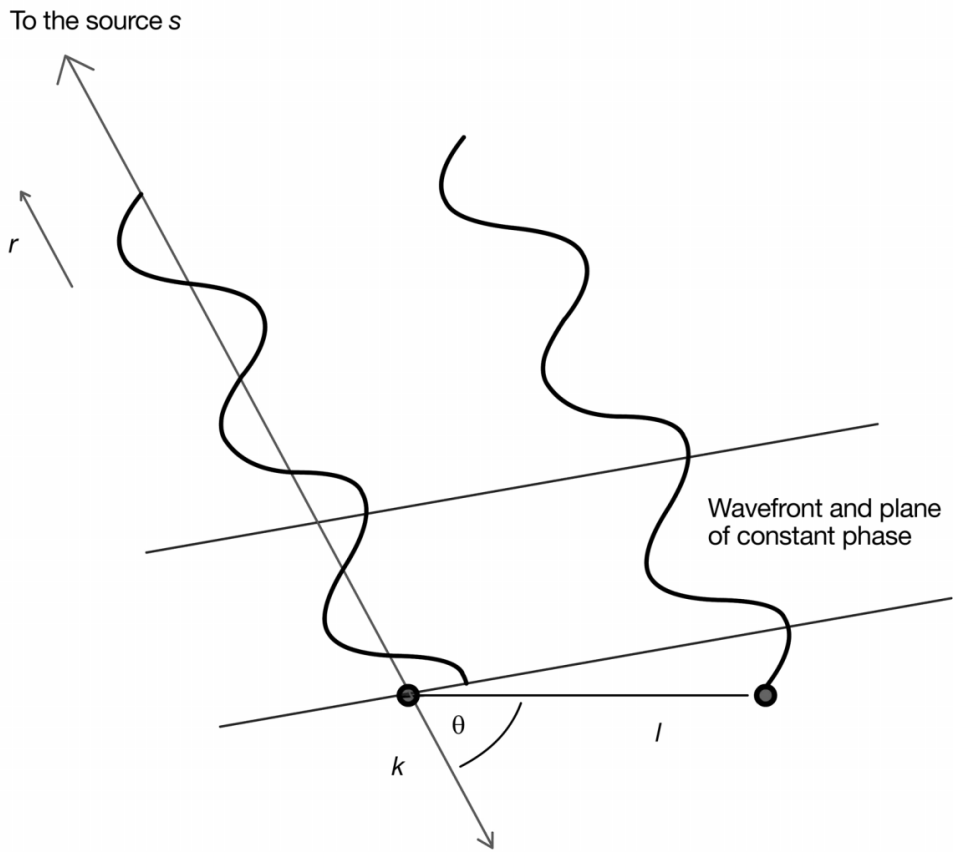

Fig. 2. The signal from the same source is received by the two antennas with a phase difference depending on the source position.

the distance $l$ of separation between the considered antennas it is possible to calculate the angle $\theta$ and consequently the horizontal position in the ionosphere.

Each antenna $a$, neglecting the environmental electromagnetic noise, will receive a composite signal of amplitude $A$ given by

$$
A_{a}(t)=\sum_{s} A_{0 a(s)} \cos \left[\left(\omega+\Delta \omega_{s}\right) t+\phi_{a s}\right]
$$

where $s$ indicates all possible sources. After the quadrature sampling the signal the two following discrete-time sequences $I_{a}$ (in-phase) and $Q_{a}$ (in-quadrature) will be obtained

$$
\begin{aligned}
& I_{a}\left(\tau_{n}\right)=\sum_{s} A_{0 a(s)} \cos \left[\Delta \omega_{s}\left(\tau_{n}\right)+\phi_{a s}\right] \\
& Q_{a}\left(\tau_{n}\right)=\sum_{s} A_{0 a(s)} \operatorname{sen}\left[\Delta \omega_{s}\left(\tau_{n}\right)+\phi_{a s}\right]
\end{aligned}
$$

where $\tau_{n}$ is the sampling time interval. If the sampling is performed at exactly the time period $\tau_{n}$ of the carrier wave $\omega$, the sampling acts like a filter rejecting the carrier $\omega$ and the two sequences will contain only the Doppler shift $\Delta \omega_{s}$. Amplitude $A(\tau 1)$ and phase $\phi(\tau 1)$ of the signal at a given time $\tau 1$ are

$$
\begin{gathered}
A=\sqrt{I_{a}(\tau 1)^{2}+Q_{a}(\tau 1)^{2}} \\
\phi=\arctan \left[Q_{a}(\tau 1) / I_{a}(\tau 1)\right] .
\end{gathered}
$$

The two discrete-time sequences $I_{a}$ and $Q_{a}$ are the input of the algorithm the performs a complex Fast Fourier Transform, FFT (Oppenheim, 1999).

The FFT of the $N$ samples (where $N$ is a power of 2) can be written as

$$
F_{a}(d)=\sum_{n=-N / 2}^{N / 2-1} f_{a}(n) e^{-i \frac{2 \pi}{N} d \cdot n}
$$

where $n$ is an index that runs from $-N / 2$ to $N / 2$, $d$ is a dummy index to perform the operation, and $f_{a}(n)$ is

$$
f_{a}(n)=I_{a}\left(\tau_{n}\right)+i Q_{a}\left(\tau_{n}\right) .
$$


The spectral analysis (complex FFT) furnishes $d=N$ couple of values representing the discrete frequencies and the related phases. The frequency resolution is $\delta \omega$, being $\delta \omega$ equal to $2 \pi / T$ and $T$ the time length of the segment of the signal considered (typically less than $20 \mathrm{~s}$ according with the time coherence of the ionosphere). The highest frequency is related to Nyquist sampling theorem and the $d \cdot \delta \omega$ is the highest Doppler shift range. It is worth applying a tapering function (Hanning or others) to the discrete and finite time sequences to avoid the ringing six $(x) / x$ after the spectral analysis.

So as a result of the complex FFT for each of the 4 antennas $N$ spectral density values $A$ and an associated time independent phase $\phi$ will be obtained. Even if negative values of the frequencies have no physical meaning, in this case they represent sources that are moving in the direction of $r$ while positive values of the spectral components are related to the incoming sources. These measurements are generally affected by environmental radio noise or interferences produced by other HF stations. So to evaluate the direction of $\boldsymbol{k}$ vector from eq. (2.5) a statistical anlysis must be performed (Scali, 1993).

\section{Radial velocity and determination of the Doppler sources}

In general the observed shift for a sounding performed at a known angular frequency $\omega$, apart from a constant factor, is the time derivative of the phase path $P$ of the radio wave

$$
\Delta \omega=\frac{-\omega}{2 c} \frac{d P}{d t} .
$$

The time derivative of the phase path depends on two terms (Dyson, 1975)

$$
\frac{d P}{d t}=p r \cdot V+\int_{0}^{r} \frac{\partial \mu}{\partial t} \cos \alpha d r
$$

where $\mu$ is the refractive index and $\alpha$ is the angle between the wave normal and the ray direction (null for isotropic medium), $V$ is the real velocity of the reflector and pr the unit vector of the wave normal at the reflection point. Hence the quantity observed is the apparent velocity,
$V_{\text {app }}$, because the time derivative of the refractive index has to be considered. If the electron density changes in time because of production or loss (especially during down and dusk) it is not possible to neglect the second term in the above equation. For that the measured velocities are not real, i.e. not only dependent on the moving reflector.

When performing ionospheric drift measurements the second term contribution on the relation (3.2) must be considered and only in stationary electron density condition are the observed velocities of the Doppler point sources related to the plasma motion. In this case, the radial velocity of each point sources comes directly from relation (1.1)

$$
V_{r s}=-\Delta \omega_{s} c / 2 \omega .
$$

If a uniform plasma motion is considered by means of statistical method (Scali, 1995; Bibl, 1998) it is possible to determine the bulk motion of the plasma (drift). The Lowell Digisonde 256 uses a least-square fit procedure described in (Dozois, 1983) in which it is possible to determine the vector velocity $V$ representing a plasma drift velocity. A similar procedure is also described in the Digisonde Drift analysis manual DDA (Scali, 1993) and makes it possible to solve the position of each point source and plot the results in a so-called sky-map.

\section{Conclusions}

In this paper the theory on which the interferometric technique relies is briefly described. The transmitting antenna has a wide beam that illuminates a large area of hundreds of square $\mathrm{km}$ in the ionosphere and the signals coming from different reflection points are resolved by combining Fourier transform and interferometric techniques. The echo signal has a superimposed frequency Doppler shift, hence the information of each point source where the signal is reflected. This technique is now affordable by means of the advanced ionospheric sounders that perform both on-line analysis and post processing on the recorded signal. The Doppler Drift Analysis DDA implemented as post analy- 
sis processing in the Digisonde 256 and DPS-4 determines the Doppler shifts of signals arriving from different directions and, in some particular stationary conditions, measures the plasma drift velocity (Bibl and Reinish, 1978).

\section{REFERENCES}

BIBL, K. (1998): Evolution of the ionosonde, Ann. Geofis. 41 (5-6), 667-680.

BIBL, K. and B.W. ReINISCH (1978): The Universal Digital Ionosonde, Radio Sci., 13, 519-530.

Bibl, K., W. Pfister, B.W. Reinisch and G.S. SAles (1975): Velocities of small and medium scale ionospheric irregularities deduced from Doppler and arrival measurements, Adv. Space Res., XV, 405-411.

DAviEs, K. (1990): Ionospheric Radio (P. Peregrinus London).

DozoIs, C.G. (1983): A high frequency radio technique for measuring plasma drift in the ionosphere (University of Massachusetts Lowell Center for Atmospheric Research, Lowell, U.S.), Rep. No. 6.

Dyson, P.L. (1975): Relationship between the rate of change of the phase path (Doppler shift) and angle of arrival, J. Atmos. Terr. Phys., 37, 1151-1154.

HunsuCKER, R.D. (1991): Radio Technique for Probing the Terrestrial Ionosphere (Springer Verlag N.Y.).

OPPENHEIM, V.A., W.R. SCHAFER and J.R. Buck (1999): Discrete-Time Signal Processing, 2nd edition (New Jersey).

Scali, J. (1993): A Quality Control Package for the Digisonde Drift Analysis (DDA), Version 2.0 (University of Massachusetts Lowell, Center for Atmospheric Research).

Scali, J., B. Reinisch, C. Dozois, K. Bibl, D. Kitrosser, M. Haines and T. Bullett (1995): Digisonde Drift Analysis (University of Massachusetts Lowell Center for Atmospheric Research).

(received February 23, 2005; accepted August 29, 2005) 